\title{
Plane turbulent buoyant jets. Part 2. Turbulence structure
}

\author{
By NIKOLAS E. KOTSOVINOS \\ W. M. Keck Laboratory of Hydraulics and Water Resources, \\ California Institute of Technology, Pasadena $\dagger$
}

(Received 28 January 1976 and in revised form 9 June 1976)

The turbulence structure of a plane vertical buoyant jet in the transition state from jet-like to plume-like growth is the object of this investigation. The ambient fluid is of uniform density and motionless except for the flow induced by the jet.

An analysis of the turbulence energy equation reveals that the production of turbulent energy by the buoyancy forces relative to the production by the shear stress increases as the jet Richardson number increases, and becomes constant for a plume-like buoyant jet.

A systematic set of experiments was carried out to examine the turbulence structure for a wide range of initial Richardson numbers, extending from a value appropriate to a jet-like flow (very close to zero) to that appropriate for a plumelike flow (approximately 0.6). Fast-response thermistors and a laser-Doppler velocimeter were used to measure the buoyant jet's temperature and velocity respectively. The temperature and velocity data were recorded magnetically in digital form and subsequently processed to extract both mean and fluctuating values. The turbulence intensity and the probability density distribution of the temperature and velocity fluctuations, the maximum and minimum temperature, the intermittency, and the frequency of crossing of the hot/cold and the cold/hot interface of a buoyant jet were investigated. It was determined that the intensity of temperature and velocity fluctuations increases with increasing Richardson number. An explanation is suggested for the large-scale vortices observed in a plume.

\section{Introduction}

Although many writers, including Morton, Taylor \& Turner (1956), Morton (1959), Brooks \& Koh (1965), Abraham (1963), Batchelor (1954) and List \& Imberger $(1973,1975)$, have attempted to describe the mean flow in a turbulent buoyant jet, there have been remarkably few experimental studies of the problem. It appears that prior to the investigations that form the subject of parts 1 and 2 of this study only Rouse, Yih \& Humphreys (1952), Lee \& Emmons (1961) and Ricou \& Spalding (1961) had studied the influence of buoyancy on jet behaviour experimentally. These earlier investigations, however, were only con-

$\dagger$ Present address: School of Engineering, University of Patras, Greece. 
cerned with the time-averaged properties of the flow and did not include any observations of the turbulent fluctuations. The structure of the turbulence within a jet has apparently been studied only in the absence of buoyancy forces (Heskestad 1965; Bradbury 1965; van der Hegge Zijnen 1957). Since the forces which drive a jet and a plume are radically different (a jet is driven by the input inertia forces and a plume by the buoyancy forces), no similarity in the turbulence structure of jets and plumes can be assumed without a detailed theoretical and experimental investigation.

This paper presents the results of an experimental study of the turbulence structure in a plane turbulent buoyant jet. The results of a study of the influence of buoyancy on the mean properties of the flow were given in part 1 . Readers wishing to familiarize themselves with the experimental methods and techniques used in both of these investigations should refer to part 1 before proceeding with this paper.

\section{Analysis of the problem}

\section{Turbulence energy equation}

The turbulence energy equation for a vertical plane buoyant jet is

$$
\frac{d}{d t} \overline{\left(q^{\prime 2}\right)}=-\overline{u^{\prime} v^{\prime}} \frac{\partial \bar{u}}{\partial y}+\overline{\rho^{\prime} u^{\prime}} \frac{\rho_{a}}{\rho_{a}}-\overline{\left(u^{\prime 2}\right.}-\overline{\left.v^{\prime 2}\right)} \frac{\partial \bar{u}}{\partial x}-\frac{\partial}{\partial y} \overline{v^{\prime}\left(\frac{p^{\prime}}{\rho_{a}}+\frac{q^{\prime 2}}{2}\right)}-\text { dissipation, }
$$

where $\overline{q^{\prime 2}}=\overline{u^{\prime 2}}+\overline{v^{\prime 2}}+\overline{w^{\prime 2}}, \rho^{\prime}$ is the density difference $\bar{\rho}(x, y)-\rho(x, y, t)$ at any point $(x, y)$ in the buoyant jet at time $t, p$ is the pressure and $w$ is the velocity component orthogonal to the $u$ and $v$ components.

Consider now the flux Richardson number $R_{f}$, which is the ratio of the buoyant production (or suppression) of turbulent energy at any point $(x, y)$ to shear-stress production at the same point:

$$
R_{f}=\frac{\overline{\rho^{\prime} u^{\prime}} g}{-\rho_{a} \overline{u^{\prime} v^{\prime}} \partial \bar{u} / \partial y} .
$$

In a rising plume the vertical acceleration of a fluid element at a point $(x, y)$ should depend upon the instantaneous buoyancy force there. Since the instantaneous buoyancy force at $(x, y)$ depends upon the instantaneous density difference $\rho^{\prime}$ it follows that the velocity and density fluctuations are well correlated and that $\overline{\rho^{\prime} u^{\prime}}>0$, which implies that part of the potential energy of the buoyancy forces is transformed into turbulent energy. The turbulent energy produced by the shear-stress term $-\overline{u^{\prime} v^{\prime}} \partial \bar{u} / \partial y$ is clearly positive and therefore the flux Richardson number is positive.

The turbulent energy equation can be rewritten as

$$
\overline{\frac{d q^{\prime 2}}{d t}}=-\overline{u^{\prime} v^{\prime}} \frac{\partial \bar{u}}{\partial y}\left(1+R_{f}\right)+\text { other terms, }
$$

which clearly indicates that the production of turbulent energy by buoyancy forces is negligible in comparison with the production by shear when $R_{f} \ll 1$, but 
is important when $R_{f}=O(1)$. The mean production by shear stress is zero along the jet axis and has a maximum in the region of maximum shear. Therefore the flux Richardson number varies across a buoyant jet and cannot be used easily to provide a measure of the importance of the buoyant production. However, the dependence on $y$ can be eliminated by integration of (1) across the jet as follows:

$$
\begin{aligned}
& \int_{\text {jet }} \frac{\overline{d q^{\prime 2}}}{d t} d y=-\int_{\text {jet }} \overline{u^{\prime} v^{\prime}} \frac{\partial \bar{u}}{\partial y} d y+\int_{\text {jet }} \overline{\rho^{\prime} u^{\prime}} g d y+\text { other terms } \\
& =-\left(\int_{\text {jet }} \overline{u^{\prime} v^{\prime}} \frac{\partial \bar{u}}{\partial y} d y\right)\left(1+\bar{R}_{f}\right)+\text { other terms, }
\end{aligned}
$$

where the gross importance of the buoyant production over the shear production is given by the integrated flux Richardson number

$$
\bar{R}_{f}=\int_{\text {jet }} \overline{g \rho^{\prime} u^{\prime}} d y / \int_{\text {jet }} \rho_{a} \overline{u^{\prime} v^{\prime}} \frac{\partial \bar{u}}{\partial y} d y .
$$

For an order-of-magnitude analysis it can be assumed that

$$
\begin{gathered}
O\left(u^{\prime}\right)=O\left(v^{\prime}\right)=\epsilon U, \quad O\left(\rho^{\prime}\right)=\epsilon \Delta \rho, \quad O(\bar{u})=U, \\
O\left(\overline{\left.u^{\prime} v^{\prime}\right)}=O\left(u^{\prime}\right) O\left(v^{\prime}\right)=\epsilon^{2} U^{2}, \quad O\left(\overline{\left.\rho^{\prime} u^{\prime}\right)}=O\left(\rho^{\prime}\right) O\left(u^{\prime}\right)=\epsilon^{2} \Delta \rho U, \quad O\left(\int d y\right)=b,\right.\right.
\end{gathered}
$$

where $\epsilon<1$ and $U, \Delta \rho$ and $b$ are respectively the characteristic velocity, density and length seales across the buoyant jet. From this we obtain

$$
\bar{R}_{f}=O\left(\Delta \rho g b / \rho_{a} U^{2}\right)=O(R(x)),
$$

where $R(x)$ is the local jet Richardson number (see part 1).

Therefore the production of turbulent energy by buoyancy forces is important relative to shear production in a plume (since $R(x) \approx 0 \cdot 6$, i.e. $\bar{R}_{f}=O(1)$ ), but negligible in a jet-like flow (say $R(x) \approx 10^{-6}$, which implies $\bar{R}_{f}=O\left(10^{-6}\right)$ ).

It is worthwhile to note, however, that since for a plume the local Richardson number is a constant, the buoyant production relative to shear production becomes a constant and therefore a plume-like buoyant jet may approximate selfpreservation. In such a case it is possible to write

$$
\left.\overline{\rho^{\prime} u^{\prime}}=\overline{\left(\rho^{\prime} u^{\prime}\right.}\right)_{m} f(y / x),
$$

where the subscript $m$ denotes values on the jet axis and $f(y / x)$ is some function of $y / x$. In a rising plume the velocity and density fluctuations should be very well correlated, so that it is possible to write

$$
\left.\left.\overline{\left(\rho^{\prime} u^{\prime}\right.}\right) m \sim \beta^{\frac{1}{3}} \overline{\left(\rho^{\prime 2}\right.}\right)_{m}^{\frac{1}{2}},
$$

where $\beta^{\frac{1}{3}}$ is the cube root of the specific buoyancy flux and has the dimensions of velocity. Therefore it is possible to write for a plume

$$
\left.\int_{\text {jet }} \overline{\left(\rho^{\prime} u^{\prime}\right.} / \rho_{a}\right) g d y \sim g x \beta^{\frac{1}{3}} \overline{\left(\rho^{\prime 2}\right)_{m}^{\frac{1}{2}} / \rho_{a} .}
$$


The buoyancy flux $\rho_{0} \beta$ is written as

$$
\begin{aligned}
\rho_{0} \beta & =\lim _{t^{\prime} \rightarrow \infty}\left\{\frac{g}{t^{\prime}} \int_{0}^{t^{\prime}} \int_{\text {jet }} u(x, y, t)\left(\rho_{a}-\rho(x, y, t)\right) d y d t\right\} \\
& =\int_{\text {jet }}\left(\rho_{a}-\bar{\rho}(x, y)\right) g \bar{u}(x, y) d y+\int_{\text {jet }} \overline{\rho^{\prime}(x, y) u^{\prime}(x, y)} g d y,
\end{aligned}
$$

so that the production of turbulent energy in a cross-section by buoyancy forces is equal to the turbulent contribution to the buoyancy flux. The distributions of mean velocity $\bar{u}(x, y)$ and density difference $\Delta \bar{\rho}=\rho_{a}-\bar{\rho}(x, y)$ across a buoyant jet are approximately self-preserving, so

$$
\int_{\text {jet }}\left(\rho_{a}-\bar{\rho}(x, y)\right) g \bar{u}(x, y) d y \sim g \overline{\Delta \rho_{m}}(x) \bar{u}_{m}(x) x,
$$

where $\overline{\Delta \rho_{m}}(x)$ and $\bar{u}_{m}(x)$ are the time-averaged mean values of the density difference and velocity along the jet axis. For a plane plume $\bar{u}_{m} \sim \beta^{\frac{1}{3}}$ (see Batchelor 1954). Hence, combining (2), (3) and (4) it is possible to write for a plume

$$
A \frac{g\left(\rho_{a}-\bar{\rho}_{m}\right) x}{\rho_{0} \beta^{z}}+B \frac{g\left(\overline{\rho^{\prime 2}}\right)_{m}^{\frac{1}{2}} x}{\rho_{0} \beta^{\frac{2}{2}}}=1,
$$

for some constants $A$ and $B$. For a plane plume the non-dimensional number $g\left(\rho_{a}-\bar{\rho}_{m}\right) x / \rho_{0} \beta^{\frac{2}{3}}$ is a constant (Rouse et al. 1952; Kotsovinos \& List 1977). Therefore the non-dimensional parameter

$$
g\left(\overline{\rho^{\prime 2}}\right)_{m}^{\frac{1}{2}} x / \rho_{0} \beta^{\frac{2}{3}}
$$

should also be a constant. In the particular case when the buoyancy arises from heat, the non-dimensional parameter given by (5) is approximated by

$$
\alpha(x) g x\left(\overline{T^{\prime 2}}\right)_{m}^{\frac{1}{2}} / \beta^{\frac{2}{3}}
$$

where $\overline{T_{m}^{\prime 2}}$ is the variance of the turbulent temperature fluctuations on the axis and $\alpha(x)$ is the thermal expansion coefficient calculated at the local temperature along the jet axis (Kotsovinos 1975).

\section{Experimental results and discussion}

Details of the experimental procedure may be found in part 1, or in Kotsovinos (1975).

Turbulence structure of temperature fluctuations

Turbulence intensity. The intensity of turbulent temperature fluctuations is defined as

$$
\overline{T^{\prime 2}}(x, y)=\lim _{t^{\prime} \rightarrow \infty}\left\{\frac{1}{t^{\prime}} \int_{0}^{t^{\prime}}[T(x, y, t)-\bar{T}(x, y)]^{2} d t\right\},
$$

where $T(x, y, t)$ is the instantaneous temperature excess above the uniform ambient temperature and $\bar{T}(x, y)$ is the time-averaged mean temperature, defined as

$$
\bar{T}(x, y)=\lim _{t^{\prime} \rightarrow \infty}\left\{\frac{1}{t^{\prime}} \int_{0}^{t^{\prime}} T(x, y, t) d t\right\} .
$$

Repeatable results were obtained for a sampling time $t^{\prime} \approx 200 \mathrm{~s}$. 


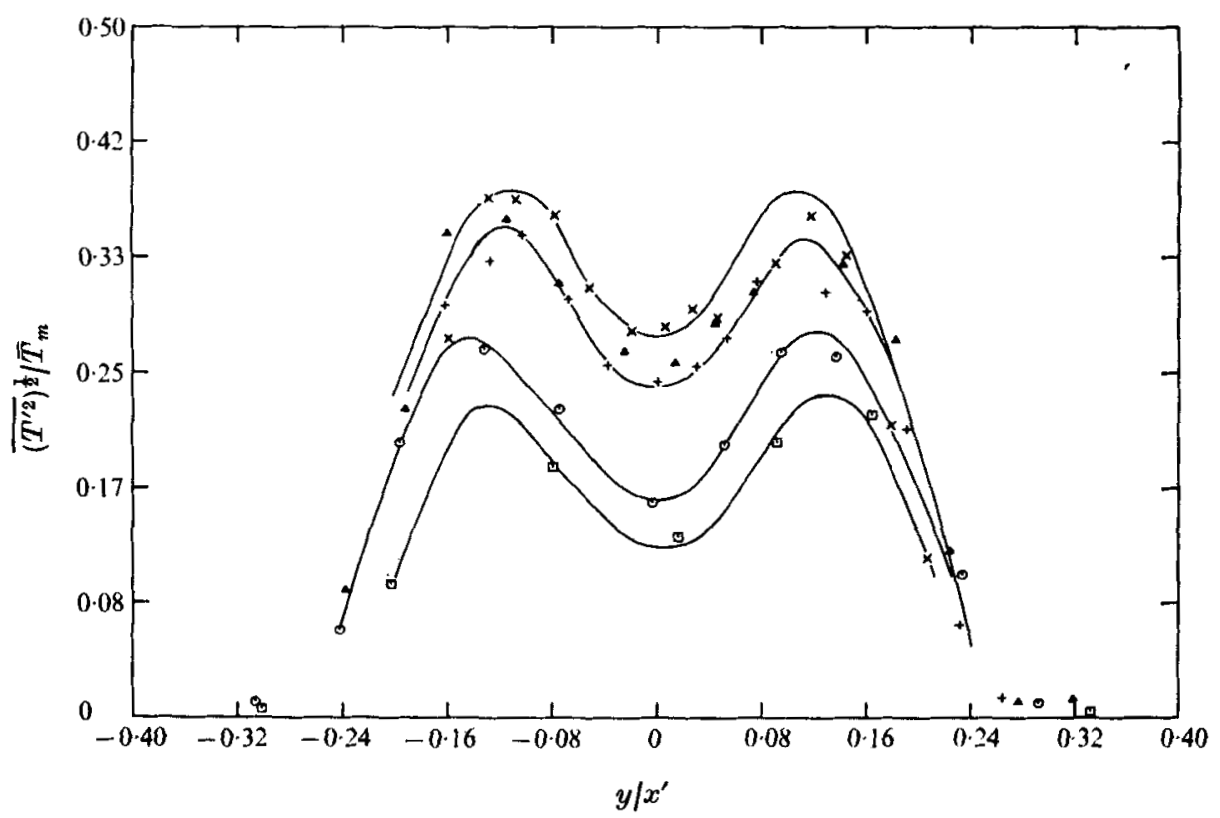

Figure 1. Profile of intensity of turbulent fluctuations of jet temperature against nondimensional distance from jet axis. Initial Richardson number $R_{0}=0.0006$. Non-dimensional distance $x / D$ from the jet orifice: []$, 6 ; 0,14 ; \Delta, 22 ;+, 30 \cdot 2 ; \times, 36$.

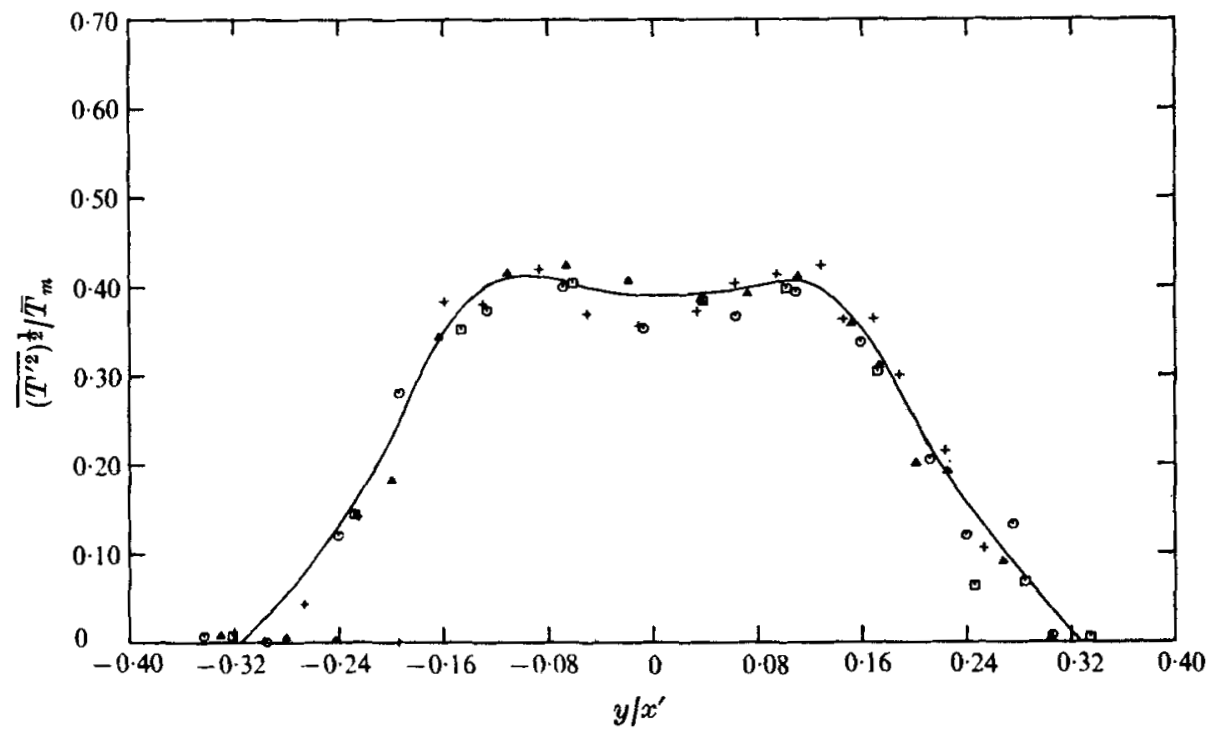

Figuas 2. Profile of intensity of turbulent fluctuations of plume temperature against non-dimensional distance from plume axis. Initial Richardson number $\boldsymbol{R}_{0}=\mathbf{0} \cdot \mathbf{2 9 5}$. Nondimensional distance $x / D$ from the plume orifice: $\square, 6 ; 0,10 ; \Delta, 14 ;+, 18$. 


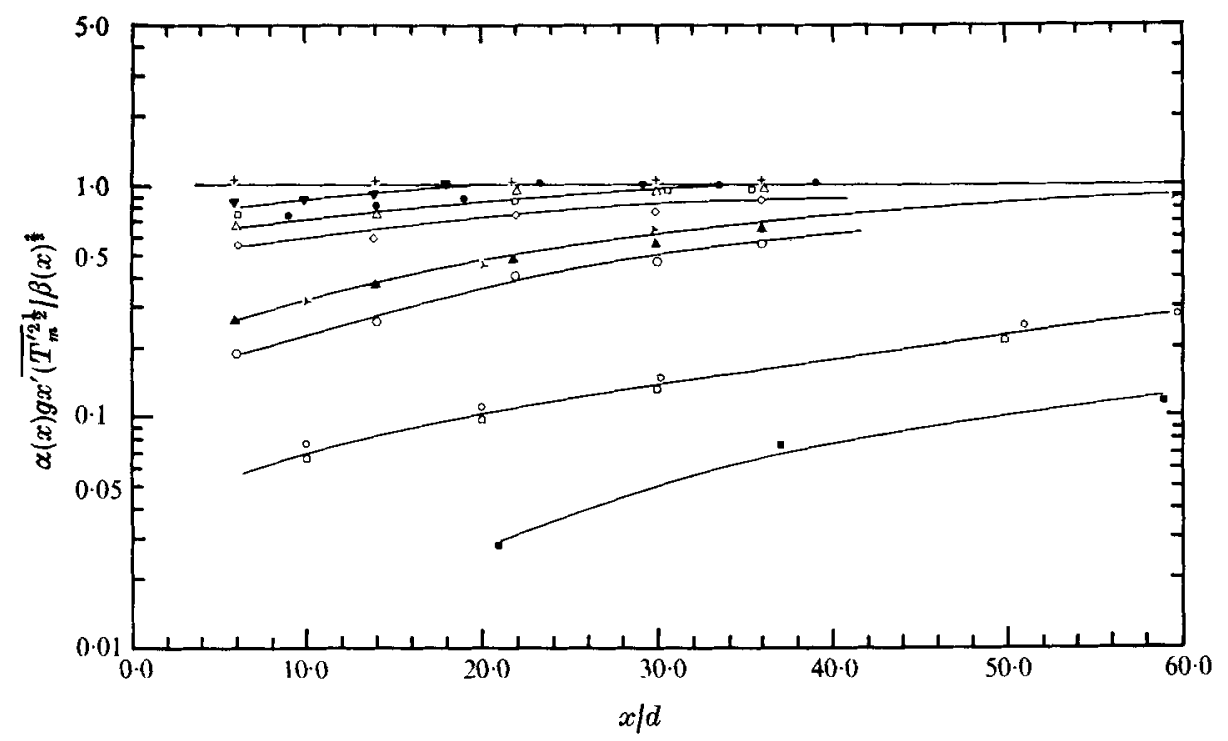

FrGURE 3. Intensity of turbulent fluctuations of temperature on jet axis as a function of non-dimensional distance from jet orifice and initial Richardson number.

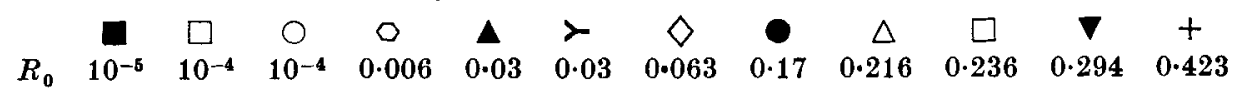

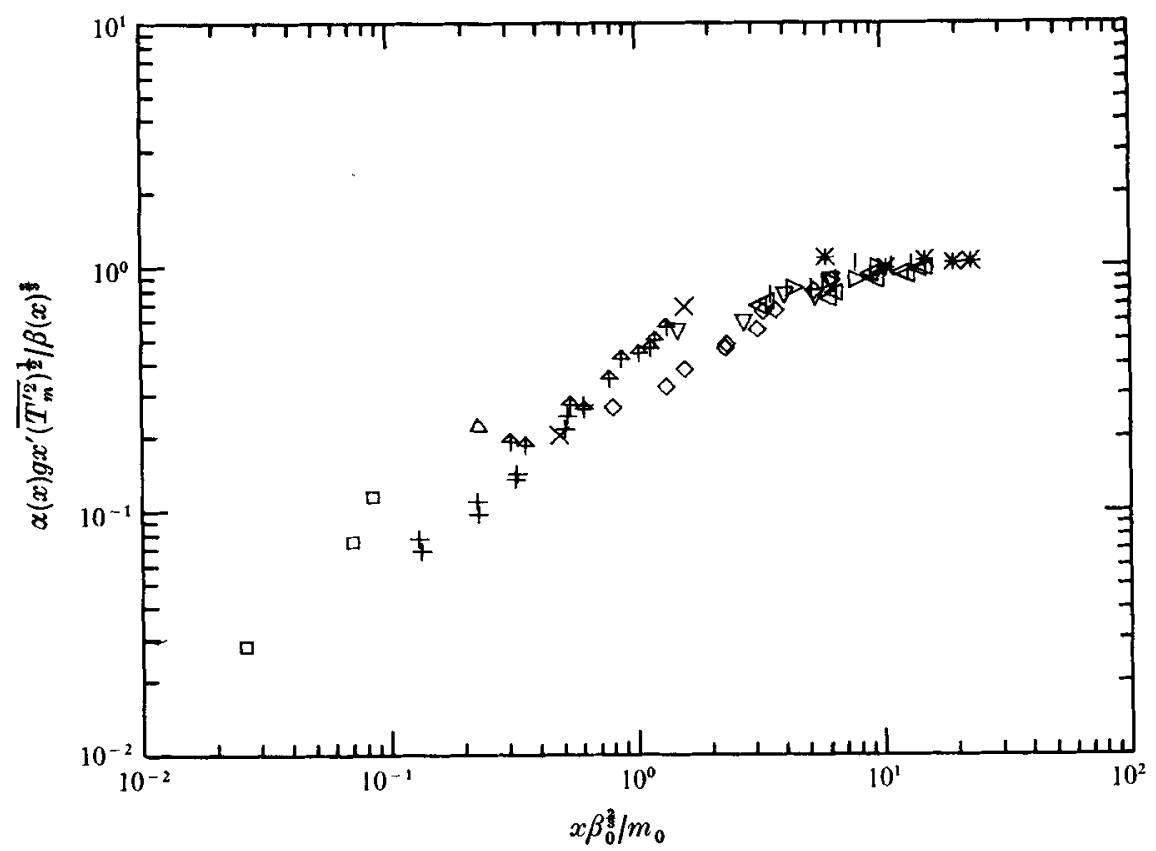

Figure 4. Normalized intensity of turbulent fluctuations in temperature on jet axis.

$\begin{array}{ccccccccccccc} & \square & \Delta & + & \times & 4 & \diamond & \nabla & \mid & \triangleleft & \triangleright & * \\ R_{0} & 0.00006 & 0.00012 & 0.0009 & 0.002 & 0.005 & 0.03 & 0.06 & 0.17 & 0.22 & 0.29 & 0.42\end{array}$




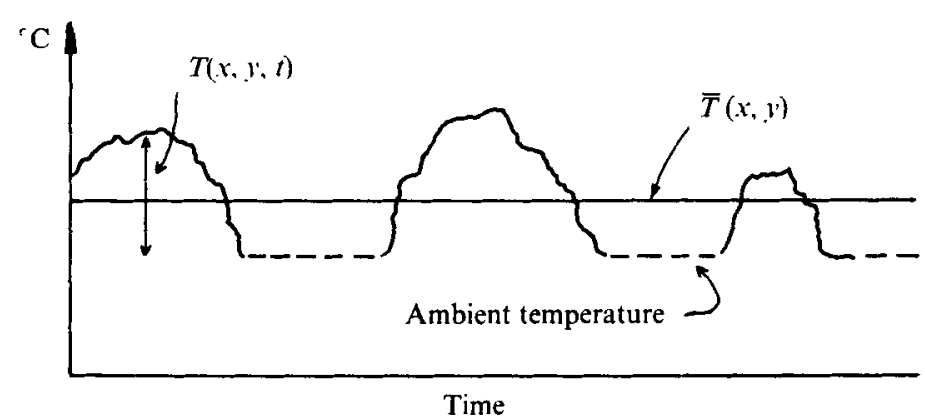

Figure 5. Sketch of an intermittent temperature signal.

The turbulence intensity $\overline{T^{\prime 2}}(x, y)$, normalized by the local mean temperature $\bar{T}_{m}(x)$ along the jet axis, is plotted versus the non-dimensional distance $y / x^{\prime}$ from the jet axis (where $x^{\prime}$ is the distance from the virtual origin of the buoyant jet) for the two representative cases of a jet-like (figure 1) and a plume-like (figure 2) flow. It is interesting to notice that the normalized turbulence intensity in a plume is larger than that in a jet. The turbulence intensity in a buoyant jet increases substantially with the distance from the jet orifice (i.e. with an increase in the local Richardson number $R(x)$ or in the parameter $x \beta_{0}^{\frac{2}{3}} / m_{0}$, where $\beta_{0}$ is the initial specific buoyancy flux and $m_{0}$ the initial specific momentum flux), until a plume-like growth is reached. In a plume the ratio of buoyant to shear production, as given by the parameter $\bar{R}_{f}$, is a constant and the normalized profile $\left.\overline{\left(T^{\prime 2}\right.}\right)^{\frac{1}{2}} / \bar{T}_{m}$ is approximately self-preserving, as predicted in $\S 2$.

The turbulence intensity $\overline{T_{m}^{\prime 2}}$ along the jet axis scaled according to $(6)$ is plotted in figure 3 versus the normalized distance $x / D$ from the jet orifice for several initial Richardson numbers $R_{0}$. It can be observed that the normalized turbulence intensity increases with increasing initial Richardson number $R_{0}$ and increasing distance $x / D$ from the exit, and that becomes a constant for a plume-like flow. The normalized turbulence intensity along the jet axis is plotted in figure 4 versus the non-dimensional parameter $x \beta_{0}^{\frac{2}{3}} / m_{0}$ (which is a measure of the relative importance of the buoyancy forces, i.e. it combines the information included in both $R_{0}$ and $x / D$, since $x \beta_{0}^{\frac{2}{3}} / m_{0}=x R_{0}^{\frac{2}{3}} / D$ ). Clearly the normalized turbulence intensity increases with an increase in the parameter $x \beta_{0}^{\frac{2}{3}} / m_{0}$ and becomes a constant for $x \beta_{0}^{\frac{2}{3}} / m_{0}>10$, i.e., regardless of how small the initial Richardson number may be, a buoyant jet will behave like a plume for $x \beta_{0}^{\frac{2}{3}} / m_{0}>$ 10.

Phase characteristics of the turbulent fluctuations. A buoyant jet is an example of intermittent flow. An intermittent temperature signal $T(x, y, t)$ recorded by a fixed probe at a point $(x, y)$ is sketched in figure 5 as a function of time $t$. During the sampling time $\tau$, there are times when the probe is surrounded by jet fluid (which is hotter than the ambient fluid; see continuous line in figure 5) and times when it is surrounded by ambient fluid (dashed line in figure 5). The turbulence intensity $\overline{T^{\prime 2}}$, as defined by ( 7$)$, is the moment of the whole signal $T(x, y, t)$, where 


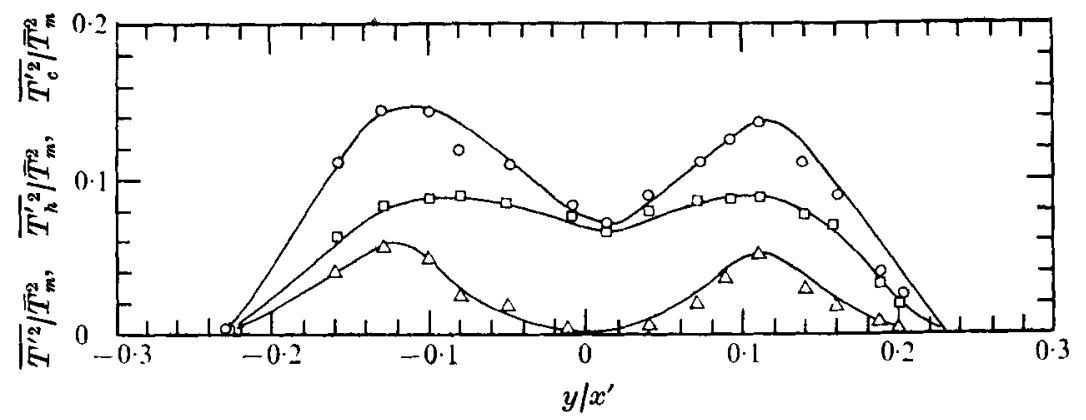

FrgURE 6. Profiles of turbulence intensities $\overline{T^{\prime 2}} / \bar{T}_{m}^{2}$ (circles), $\overline{T_{n}^{\prime 2}} / \bar{T}_{m}^{2}$ (squares) and $\overline{T_{c}^{\prime 2}} / \bar{T}_{m}^{\prime 2}$ (triangles) for a turbulent jet. Initial Richardson number $R_{0}=0 \cdot 004$, non-dimensional distance from jet orifice $x / D=\mathbf{3 6}$.

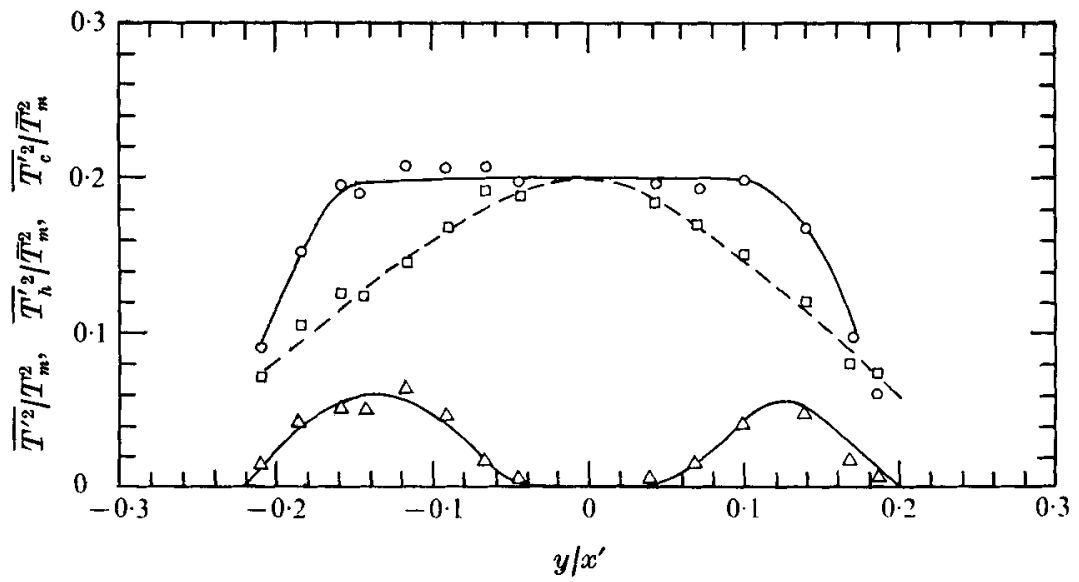

Figure 7. Profiles of turbulence intensities $\overline{T^{\prime 2}} / \bar{T}_{m}^{2}$ (circles), $\overline{T_{h}^{\prime}} / \bar{T}_{m}^{2}$ (squares) and $\overline{T_{c}^{\prime 2}} / \bar{T}_{m}^{2}$ (triangles) for a turbulent plume. Initial Richardson number $R_{0}=\mathbf{0} \cdot \mathbf{4 2 3}$, non-dimensional distance from plume orifice $x / D=30$.

$0 \leqslant t \leqslant \tau$, with respect to the mean temperature $\bar{T}(x, y)$ as defined by $(8)$. However the turbulence intensity $\overline{T^{\prime 2}}$ can be split into two distinct components:

$$
\begin{gathered}
\overline{T^{\prime 2}}=\lim _{t^{\prime} \rightarrow \infty}\left\{\frac{1}{t^{\prime}} \int_{0}^{t^{\prime}}[T(x, y, t)-\bar{T}(x, y)]^{2} d t\right\} \\
=\lim _{t^{\prime} \rightarrow \infty} \frac{1}{t^{\prime}}\left\{\int_{0}^{t^{\prime}}[T(x, y, t)-\bar{T}(x, y)]^{2}[1-I(x, y, t)] d t\right\} \\
+\lim _{t^{\prime} \rightarrow \infty}\left\{\frac{1}{t^{\prime}} \int_{0}^{t^{\prime}}[T(x, y, t)-\bar{T}(x, y)]^{2} l(x, y, t) d t\right\}, \\
I(x, y, t)= \begin{cases}1 & \text { if } T(x, y, t)>\bar{T}_{L}, \\
0 & \text { otherwise. }\end{cases}
\end{gathered}
$$

where

The temperature level $\bar{T}_{L}$ was approximately $0.05^{\circ} \mathrm{C}$ in this study. The first and the second terms on the right-hand side of (9) will be called $\overline{T_{c}^{\prime 2}}$ and $\overline{T_{h}^{\prime 2}}$ respectively. The component $\overline{T_{c}^{\prime 2}}$ can be considered as the contribution to the variance 


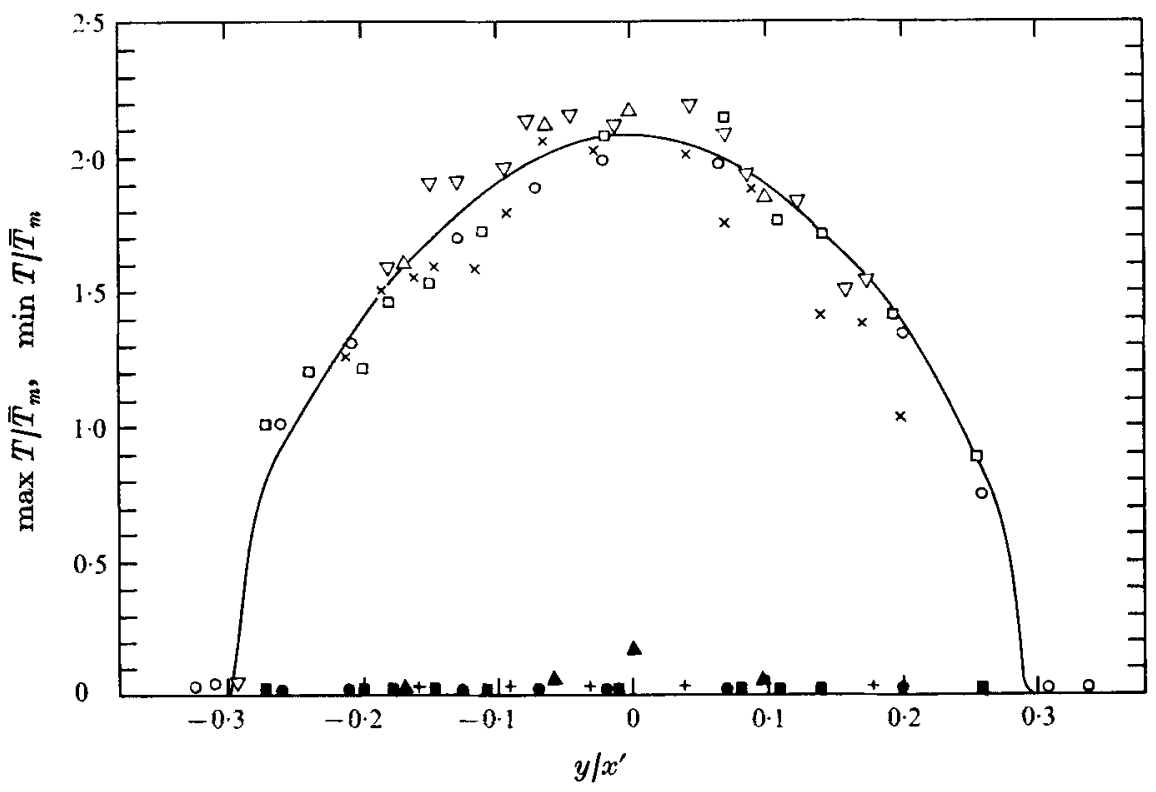

Figure 8. Maximum and minimum temperature for a turbulent plume as a function of distance from plume axis. $R_{0}=0 \cdot 423$. Open symbols, $\times, \max T / \bar{T}_{m}$; filled symbols, + , $\min T / \bar{T}_{m} . \Lambda, x / D=6 ; \bigcirc, x / D=14 ; \square, x / D=22 ;+, \times, x / D=30 ; \nabla, x / D=36$.

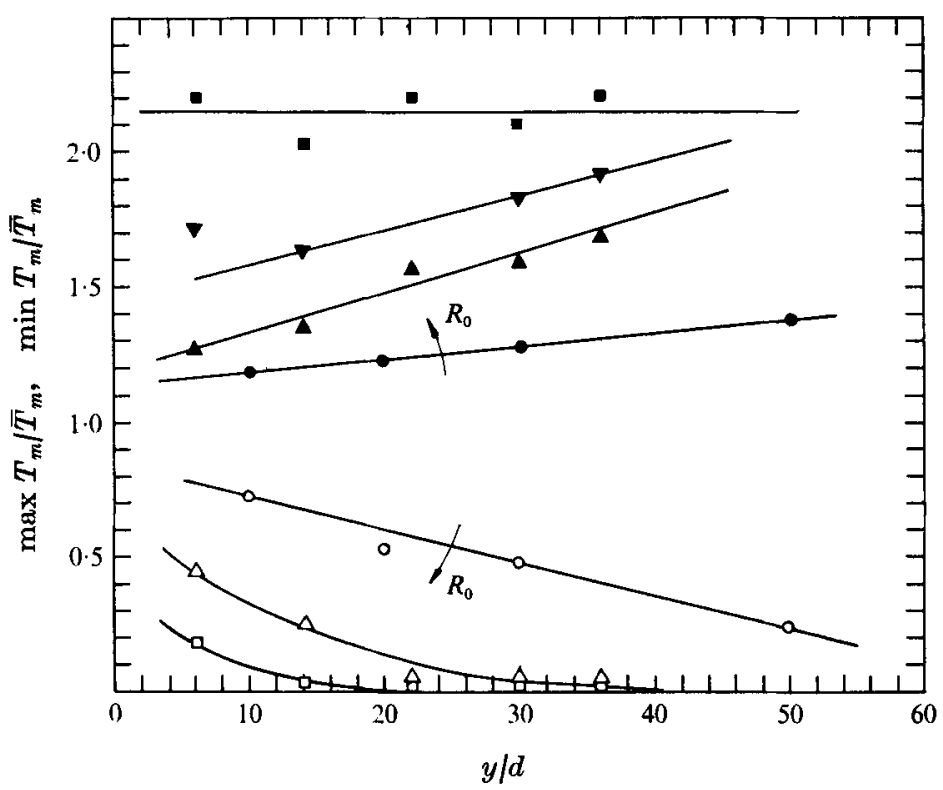

Fraure 9. Distribution of maximum and minimum temperature along jet axis as a function of initial Richardson number. Open symbols, $\max T_{m} / \bar{T}_{m}$; filled symbols, $\min T_{m} / \bar{T}_{m}$. - $R_{0}=0.0009 ; \Delta, R_{0}=0.005 ; \nabla, R_{0}=0.06 ; \square, R_{0}=0.423$. 
of the temperature generated by the intermittency of the flow and does not necessarily imply turbulence. The lateral distributions of $\overline{T^{\prime 2}}, \overline{T_{c}^{\prime 2}}$ and $\overline{T_{h}^{\prime 2}}$ are plotted in figures 6 (jet-like flow) and 7 (plume-like flow).

The two peaks in the profile of the turbulence intensity $\overline{T^{\prime 2}}$ of a jet-like flow (figure 6) are mainly generated by $\overline{T_{c}^{\prime 2}}$, i.e. by cold irrotational bursts pumped into the jet by large hot vortices, represented by temporary submergence of the thermistor in ambient fluid. Therefore in free turbulent flow, where large vortices, small-scale turbulent eddies and irrotational bursts all occur, regarding the $\overline{T^{\prime 2}}$ profile as a 'turbulence' intensity profile is evidently questionable. It can be observed from figures 8 and 9 that the normalized profile $\overline{T_{c}^{\prime 2}} / \overline{T_{m}^{\prime 2}}$ is approximately the same for both jet-like and plume-like flows, but that the $\overline{T_{h}^{\prime \prime}} / \overline{T_{m}^{\prime 2}}$ profile is larger in a plume than in a jet.

Profile of maximum and minimum temperature. The maximum temperature $\max T$ is defined by

$$
\max T=\max _{0<t<\tau} T(x, y, t)
$$

where $\tau$ is the sampling time, while the minimum temperature $\min T$ is defined by

$$
\min T=\min _{0<t<\tau} T(x, y, t) .
$$

The maximum and minimum temperatures occurring at a given point $(x, y)$ during a sampling time $\tau$, made non-dimensional using the mean centre-line temperature $\bar{T}_{m}(x)$, are plotted in figure 8 for the case of a plume-like flow. The boundaries of the jet can be defined as the distance $y / x^{\prime}$ at which the profile of the maximum recorded temperature drops abruptly to zero.

Clearly there is a finite probability of finding jet fluid at the jet boundaries with temperature approximately equal to the mean temperature on the jet axis. This suggests that for some applications the buoyant jet should be viewed from a probabilistic rather than a deterministic point of view.

A conclusion from figure 9 is that the non-dimensional centre-line maximum temperature $\max T_{m} / \bar{T}_{m}$ and the non-dimensional centre-line minimum temperature $\min T_{m} / \bar{T}_{m}$ depend on the local Richardson number $R(x)$. Specifically, $\max$ $T_{m} / \widehat{T}_{m}$ increases with $R(x)$ and tends to an asymptotic value equal to $2 \cdot 5$ for $R(x) \approx R_{p}$, while min $T_{m} / \bar{T}_{m}$ decreases with increasing $R(x)$ and becomes zero for $R(x) \approx R_{p}$. This is consistent with the previous result, i.e. that the turbulence intensity increases with $R(x)$. The interesting result $\min T_{m} / \bar{T}_{m}=0$ for a plume implies that the intermittency of the temperature at the centre of the plume is not unity, and cold ambient fluid ean reach the axis of the plume.

Intermittency factor. The intermittency factor $\gamma(x, y)$ is defined as

$$
\gamma(x, y)=\lim _{t^{\prime} \rightarrow \infty}\left\{\frac{1}{t^{\prime}} \int_{0}^{t^{\prime}} I(x, y, t) d t\right\}
$$

The lateral distribution of the intermittency factor is plotted in figure 10 .

The intermittency factor along the axis of the plume-like flow was found to be less than one (approximately $0 \cdot 98-0.99$ ), which is consistent with the minimum 


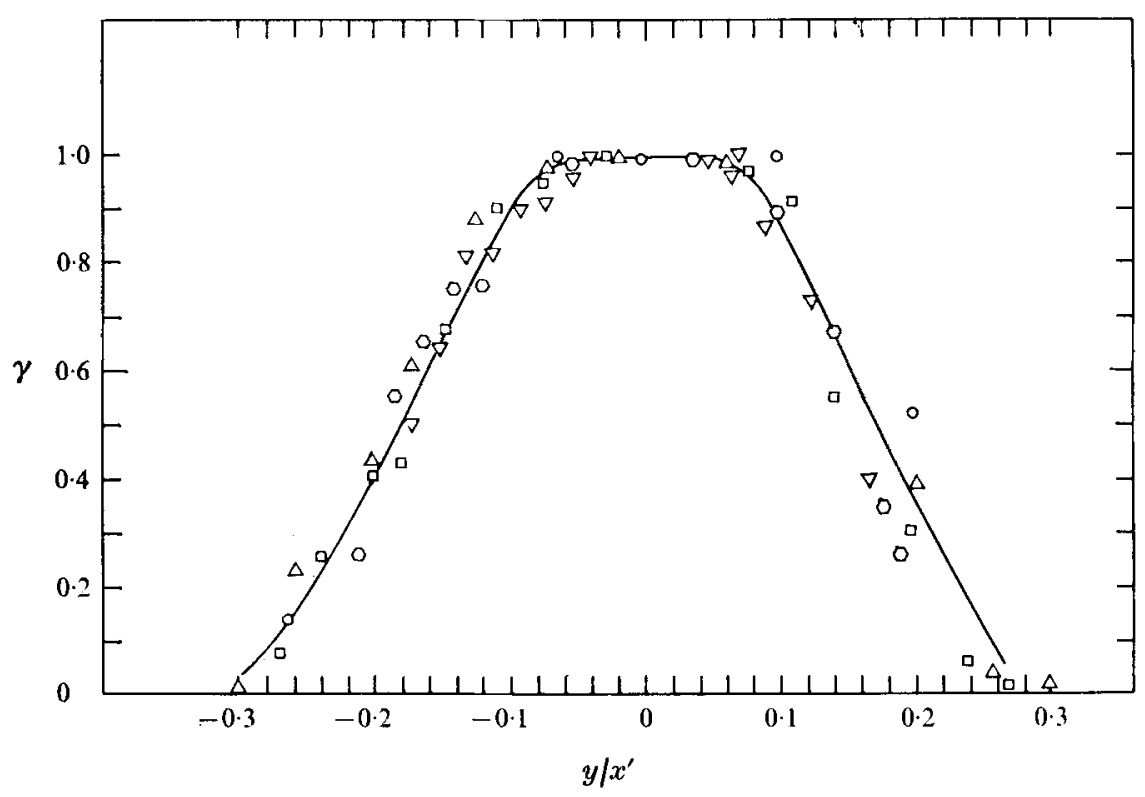

Figure 10. Temperature intermittency for a turbulent plume against non-dimensional distance from plume axis. Initial Richardson number $R_{0}=0 \cdot 423$. Non-dimensional distance $x / D$ from plume orifice: $O, 6 ; \triangle, 14 ; \square, 22 ; 0,30 ; \nabla, 36$.

recorded temperatures (figure 9) and with the flow visualization (figure 18, plate 1), which indicates the existence of large vortices extending across the whole width of the plume. It should be noted that the intermittency factor depends on the size of the probe, which limits the size of the smallest detectable 'cold' eddy (see also Shaughnessy 1975, p. 102), and on the threshold level $\bar{T}_{L}$ (in this study $\bar{T}_{L} \approx 0.05{ }^{\circ} \mathrm{C}$ and $\left.T_{L} / \bar{T}_{m}<0.02\right)$.

Frequency of crossing of hot/cold interface. The intermittent signal sketched in figure 5 contains two basic components, one above the temperature of the ambient fluid (associated with the passage of hot large-scale vortices, usually called 'bursts') and another at the ambient temperature. The frequency $f_{R}$ of the bursts can be estimated by measuring the frequency of crossing of the hot/cold interface. Therefore one way to find $f_{R}$ is to construct a function $M\left(x, y, t_{i}\right)$ such that

$$
M\left(x, y, t_{i}\right)=\left\{\begin{array}{lll}
0 & \text { if } & {\left[I\left(x, y, t_{i}\right)+I\left(x, y, t_{i+1}\right)\right]=0 \text { or } 2} \\
1 & \text { if } & {\left[I\left(x, y, t_{i}\right)+I\left(x, y, t_{i+1}\right)\right]=1}
\end{array}\right.
$$

where $t_{i}, i=1,2, \ldots, N$, is the time (in seconds) of the $i$ th sample, conditionally recorded during the sampling time $t_{N}$.

Then the frequency of bursts is given by

$$
f_{R}=\frac{\sum_{i=1}^{N} M\left(x, y, t_{i}\right)}{2\left(t_{N}-t_{1}\right)},
$$

and is plotted in figure 11. From the intermittency factor $\gamma$ and the frequency of bursts $f_{R}$ at a point $(x, y)$ the average duration (in seconds) of a 'hot' burst (time 


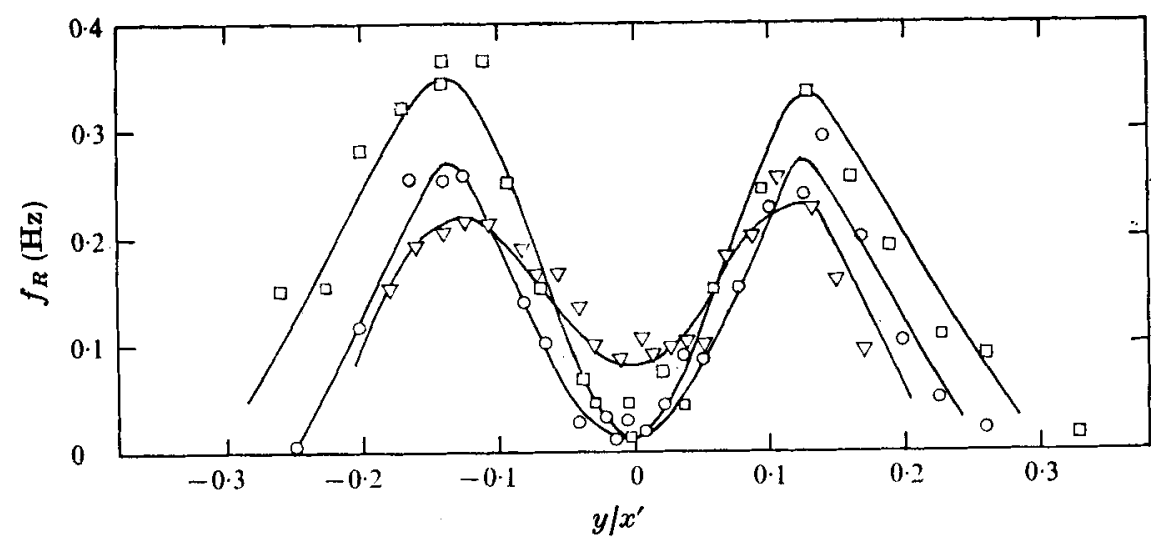

FIGURE 11. Profile of frequency of hot/cold, cold/hot interface crossing for a turbulent plume. Initial Richardson number $R_{0}=0 \cdot 23$. Non-dimensional distance $x / D$ from plume orifice: $\square, 22 ; 0,30 ; \nabla, 36$.

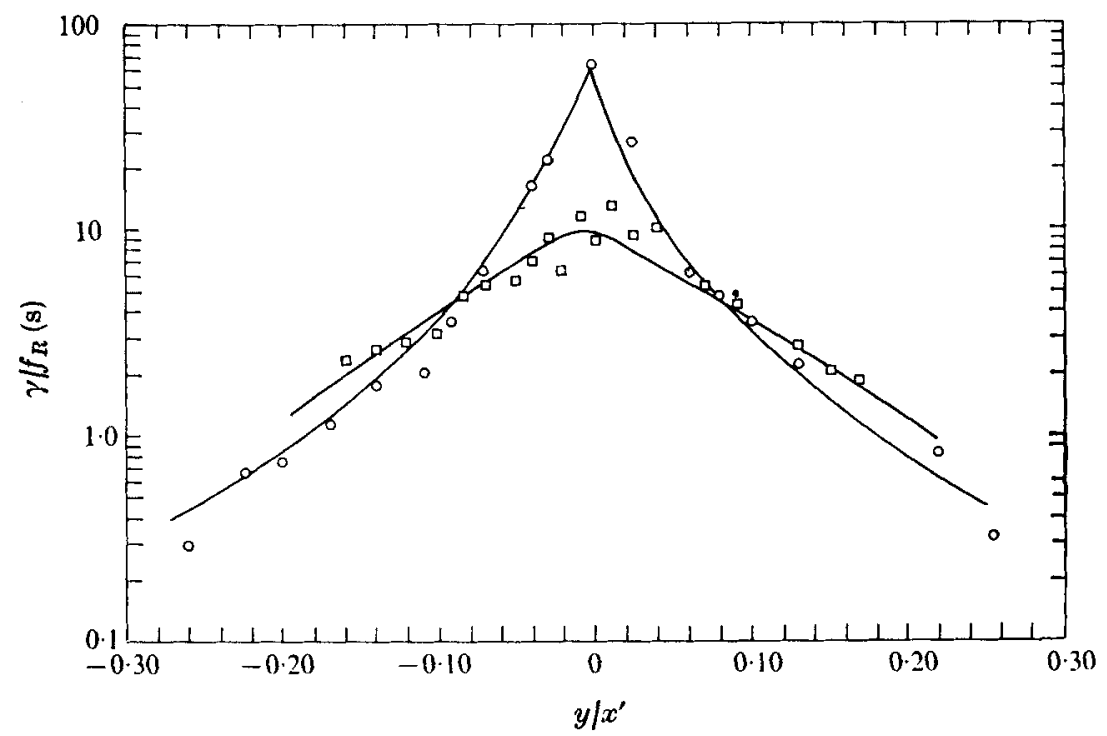

Figure 12. Average duration of hot bursts in a turbulent plume. Initial Richardson number $R_{0}=0.23$. Non-dimensional distance $x / D$ from plume orifice: $\bigcirc, 22 ; \square, 36$.

between two successive cold/hot, hot/cold interface crossings) can be found to be $\gamma(x, y) / f_{R}(x, y)$, and is plotted in figure 12 .

It should be noted that the nature of these measurements relies essentially on the form of the test function $I(x, y, t)$, which depends on the threshold level $\bar{T}_{L}$. In this investigation $\bar{T}_{L} \approx 0.05^{\circ} \mathrm{C}$. The absolute value of $f_{R}$ depends on the value of the threshold level $\bar{T}_{L}$ chosen, but a systematic investigation of this dependence was not made. The important point is that keeping $\bar{T}_{L}$ constant for a particular experiment resulted in the same structure being revealed. 

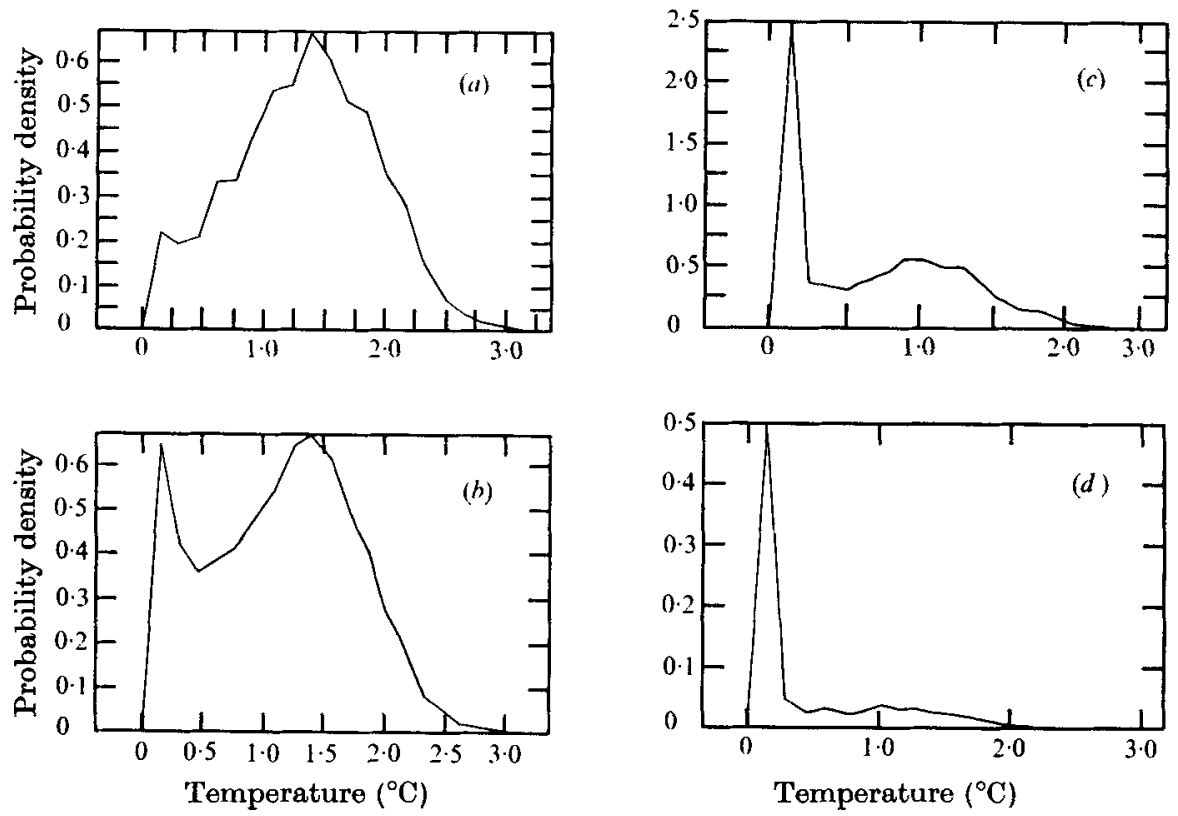

Figure 13. Probability density of the temperature of a turbulent plume. Initial Richardson number $R_{0}=0.17$. Non-dimensional distance $y / x^{\prime}$ from plume axis: (a) 0.005 ; (b) $0.055 ;(c) 0.098 ;(d) 0.148$.

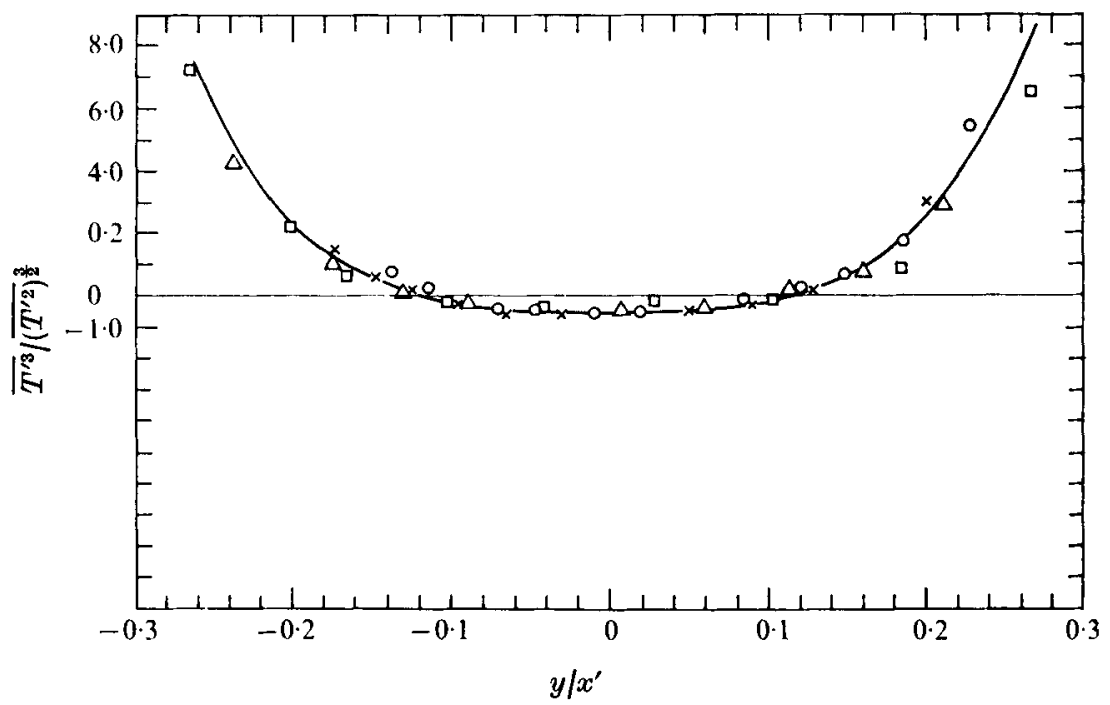

Figure 14. Skewness factor of temperature fluctuations of a buoyant jet as a function of distance from the jet axis. Initial Richardson number $R_{0}=0 \cdot 03$. Non-dimensional distance from jet orifice: $\square, 14 ; \triangle, 22 ; \times, 30 ; 0,36$. 


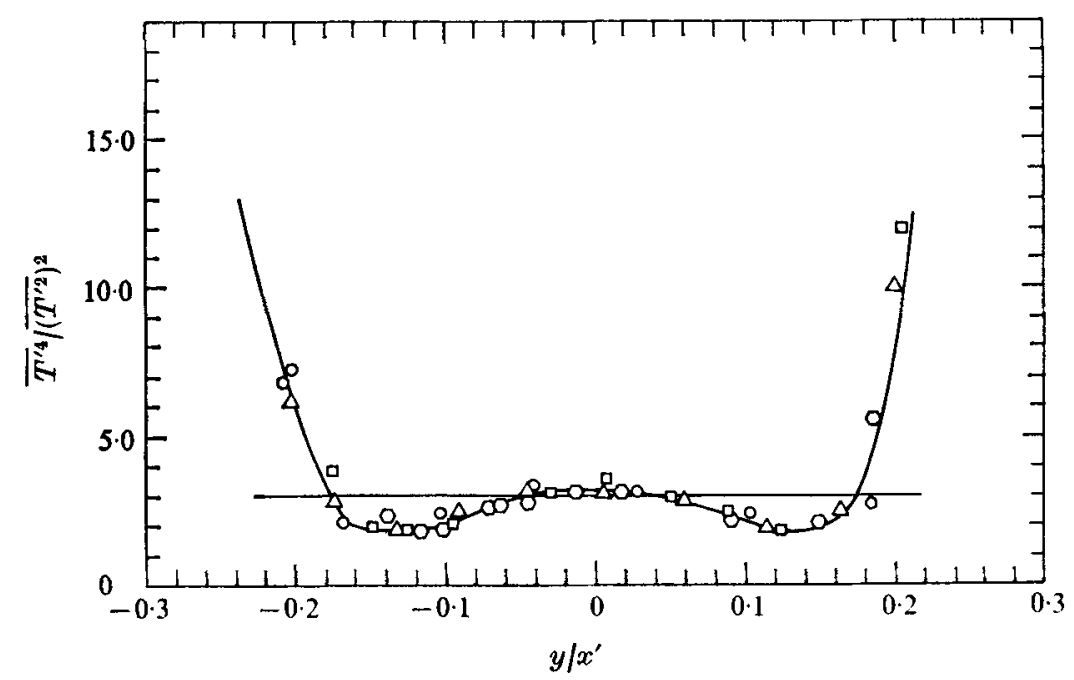

FIgURE 15. Flatness factor of temperature fluctuations of a buoyant jet as a function of distance from jet orifice. Initial Richardson number $R_{0}=0.029$. Non-dimensional distance from jet orifice: 0,$14 ; \triangle, 22 ; \square, 30 ; 0,36$.
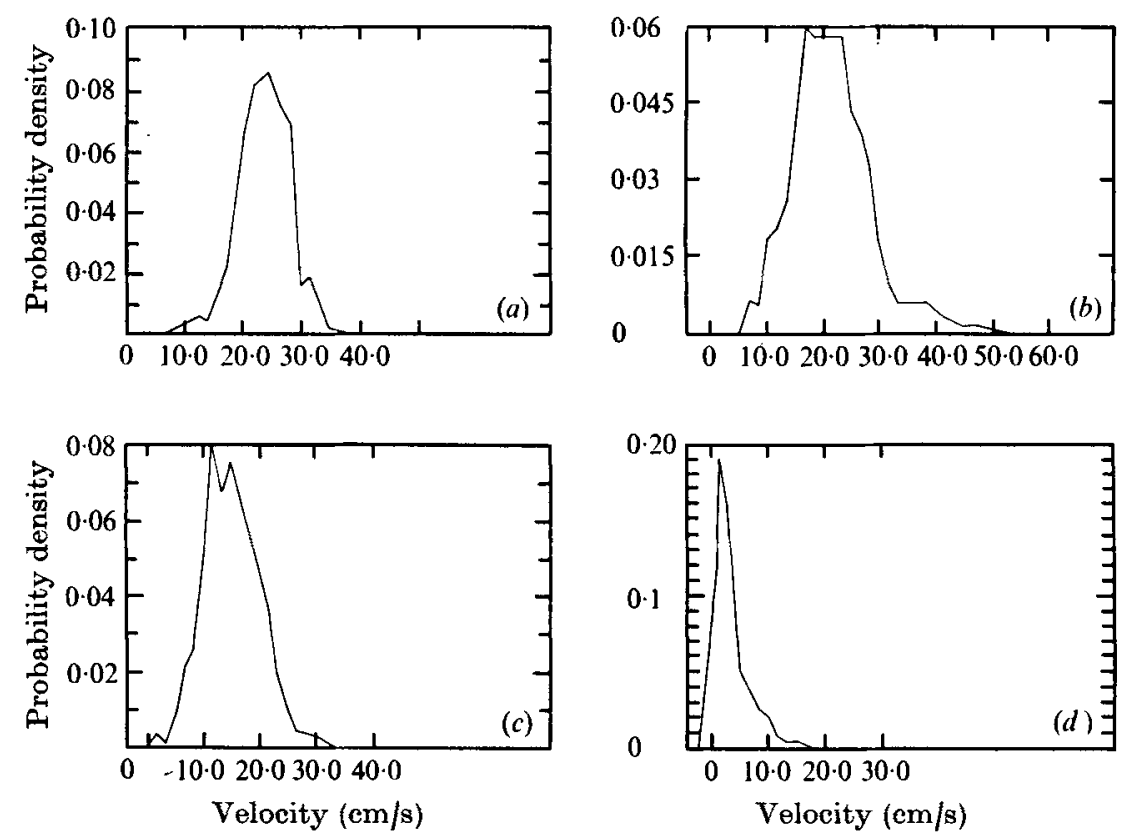

Figure 16. Probability density of the velocity of a turbulent jet $\left(R_{0}=0\right)$. Non-dimensional distance $y / x^{\prime}$ from jet axis: $(a) 0.021 ;(b) 0.046 ;(c) 0.088 ;(d) 0.154$.

The probability density distribution of temperature fluctuations. The probability density distribution of the conditionally sampled instantaneous temperature is plotted in figures $13(a)-(d)$ for four typical positions across a plume-like flow. The area under the peak in the distribution that occurs near zero temperature increment is approximately equal to the fraction of the time that the recorded tem- 


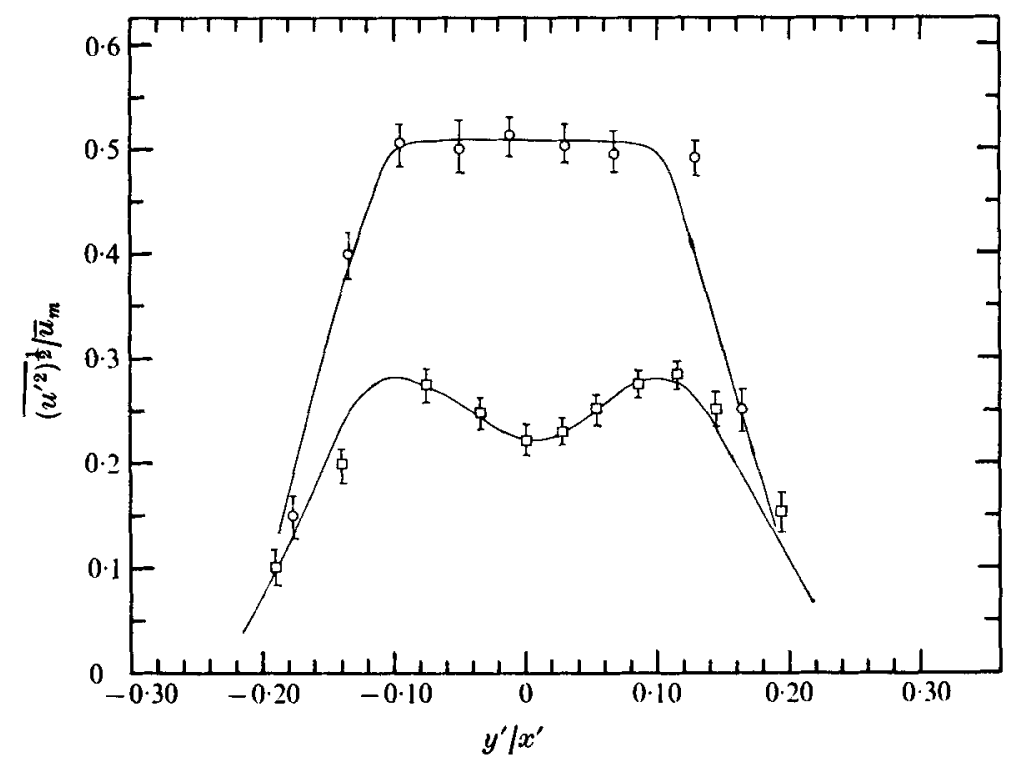

Figure 17. Root-mean-square level of turbulent velocity fluctuations in a plume (circles) and a jet (squares).

perature is equal to the ambient temperature. Since the whole area under the probability density distribution is unity, the area under this peak is approximately $1-\gamma(x, y)$.

Finally, it can be seen that if the peak in the distribution, which is generated by large eddies, is removed, then the remaining distribution looks quite symmetric, which implies that the smaller scales of turbulence may be isotropic.

Skewness and flatness factor of temperature fluctuations. The skewness factor, defined as $\left.\overline{T^{\prime 3}} \overline{T^{\prime 2}}\right)^{\frac{3}{2}}$, is plotted in figure 14 and the flatness factor, defined as $\overline{T^{\prime 4}} /\left(\overline{\left.T^{\prime 2}\right)^{2}}\right.$, is plotted in figure 15. For a Gaussian curve the skewness factor is zero and the flatness factor $3 \cdot 0$, values which are approximately attained along the jet axis.

\section{Turbulence structure of the axial velocity fluctuations}

The signal from the laser-Doppler velocimeter was processed using a spectrum analyser (see Kotsovinos 1975). Therefore an analog signal in real time proportional to the instantaneous velocity was not available. However, the probability density distribution and the turbulence intensity could be obtained, but the intermittency or the frequency of the turbulent/non-turbulent velocity interface could not be easily obtained.

The probability density distribution of velocity is plotted in figure 16 for a jet-like flow. The occurrence of negative velocities (i.e. velocities with direction opposite to the jet flow) was observed on the oscilloscope of the spectrum analyser; an estimate of their magnitude and probability can be obtained from the figures. One possible mechanism for the generation of such negative velocities is the production of large vortices which could contain negative velocities larger than 


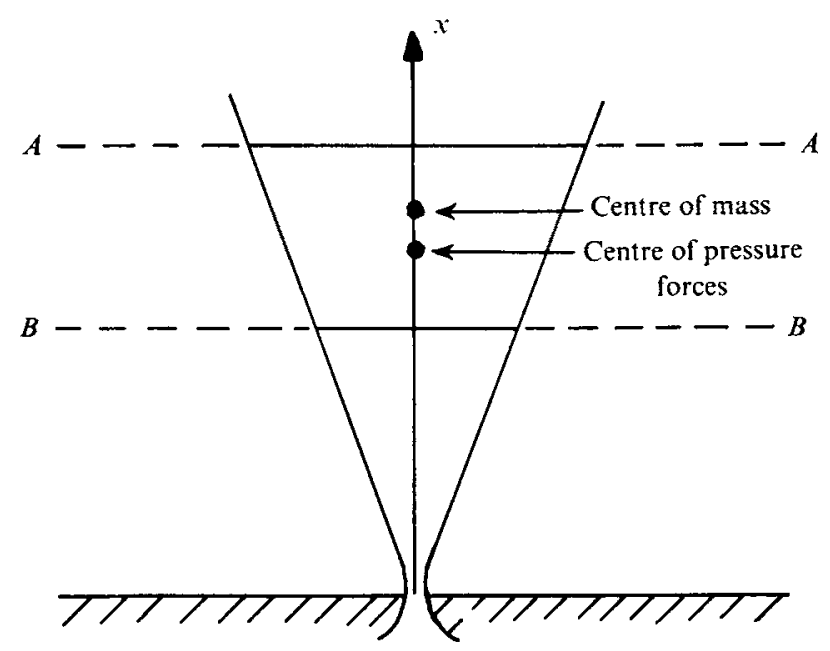

FrguRe 21. Hypothetical instantaneous picture of a plume.

their advection velocity. Another possible mechanism, valid only for plane jetlike flow, is the reversed flow component of the induced flow, as will be seen in the flow-visualization experiments to be discussed below (figure 20, plate 3).

The profile of turbulence intensity $\overline{u^{\prime 2}}$ is plotted in figure 17 for a jet-like and a plume-like flow and it can be seen that this confirms the results obtained from temperature measurements.

\section{Flow visualization}

Figures 18 and 19 are short time $(0.01 \mathrm{~s})$ and medium time $(2 \mathrm{~s})$ exposure photographs of a turbulent plane plume, whereas figure 20 (plate 3 ) is a medium time exposure ( $2 \mathrm{~s}$ ) photograph of a turbulent plane jet (without buoyancy). The difference in the induced flow field can readily be appreciated by comparing the dye lines in figures 19 and 20 . It is interesting to observe the large-scale vortices in the plume in figure 18. A detailed stability analysis will not be presented here, but a basic mechanism which could produce these large-scale vortices is as follows. Assume that at some time $t_{0}$ a plume achieves the configuration of figure 21 and consider the volume $V_{A B}$ which is included between the cross-sections $A A$ and $B B$. Now the temperature on the plume axis decreases and the density increases as the distance $x$ from the plume orifice increases. Hence the centre of mass of the volume $V_{A B}$ will be above the centre of the pressure forces. Two external forces are acting on the mass $m_{A B}$ of the volume $V_{A B}$ :(i) the gravitational force, with absolute value

$$
G=m_{A B} g
$$

which acts at the centre of mass, and (ii) the Archimedean force, with absolute value

$$
R=V_{A B} \rho_{a} g,
$$

which acts at the centre of pressure forces. If the flow depicted in figure 21 is slightly perturbed then the situation depicted in figure 22 is obtained, from which 


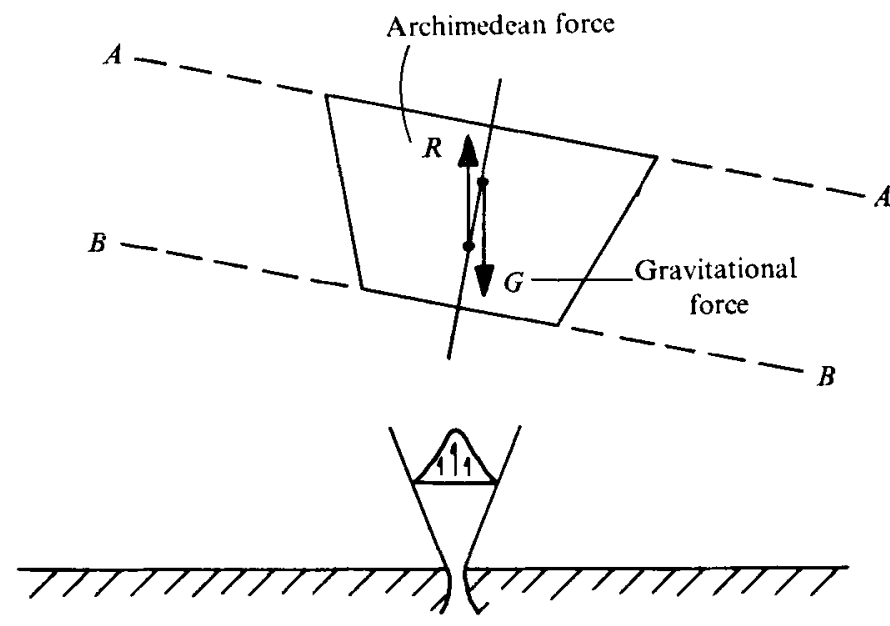

Figure 22. Schematic diagram explaining the production of large vortices in a plume.

we can observe that the moment between the forces $R$ and $G$ tends to overturn the volume $V_{A B}$. Such a mechanism could lead to the large-scale vortices which are observed in a plume.

These large vortices extend across the whole width of the plume (therefore their characteristic length scale increases like $x$ ), are fairly closely two-dimensional and appear to be different in structure from the large vortices in a jet, which are smaller and do not reach (at least visually) the central jet region. The important role of vortex formation as the basic mechanism for the entrainment in free shear flows has been recognized only recently (see Papailiou \& Lykoudis 1974; Laufer 1975). Large-scale 'hot' vortices engulf ambient 'cold' irrotational fluid, increase in size and then suddenly break down into small-scale hot and cold masses, which are subsequently mixed by turbulent and molecular diffusion.

It is known that the entrainment coefficient in a plume is twice the entrainment coefficient in a jet (part 1). Since the scale of the large vortices is much smaller in a jet than in a plume, it can be argued that the plume achieves larger entrainment by increasing the size of the large-scale vortices.

The subject of this paper is taken in part from the author's Ph.D. thesis, submitted to the California Institute of Technology. The author is grateful to Professor E.J.List for many constructive and enlightening discussions. The financial support of the National Science Foundation (Grant ENG75-02985) is also gratefully acknowledged.

\section{REFERENCES}

Aвrayam, G. 1963 Jet diffusion in stagnant ambient fluid. Delft Hydraul. Lab. Publ. no. 29.

Batchelor, G. K. 1954 Heat convection and buoyancy effects in fluids. Quart. J. Roy. Met. Soc. 80, 339-358.

Bradbury, L. 1965 The structure of the self-preserving turbulent plane jet. J. Fluid Mech. .33, 31-64. 
Brooks, N. H. \& KoH, R. C. Y. 1965 Discharge of sewage effluent from a line source into a stratified ocean. 11th Cong. Int. Assoc. Hydraul. Res, paper 2.19.

HegGe ZiJNen, B. G. vaN Der 1957 Measurements of turbulence in a plane jet of air by the diffusion method and by the hot-wire method. Appl. Sci. Res. 7, 292-313.

Heskestad, G. 1965 Hot wire measurements in a plane turbulent jet. J. Appl. Mech. 33, 721-734 (erratum, Sept. 1966, p. 710).

Kotsovinos, N. E. 1975 A study of the entrainment and turbulence in a plane buoyant jet. W. M. Keck Lab. Hydraul. Water Res., Caltech Rep. KH-R-32.

Kotsovinos, N. E. \& LIST, E. J. 1977 Turbulent buoyant jets. Part 1. Integral properties. J. Fluid Mech. 81, 25-44.

LAUfER, J. 1975 New trends in experimental turbulence research. Ann. Rev. Fluid Mech. 7, 307-326.

LEE, S. L. \& Emmons, H. W. 1961 A study of natural convection above a line fire. $J$. Fluid Mech. 11, 353-368.

LIST, E. J. \& IMBERGER, J. 1973 Turbulent entrainment in buoyant jets and plumes. J. Hydraul. Div. A.S.C.E. 99, 1461-1474.

LIST, E. J. \& IMBERGER, J. 1975 Concluding discussion of paper 'Turbulent entrainment in buoyant jets and plumes.' J. Hydraul. Div. A.S.C.E. 101, 617-620.

Monton, B. R. 1959 Forced plumes. J. Fluid Mech. 5, 151-163.

Morton, B., TAylor, G. I. \& TuRner, J. S. 1956 Turbulent gravitational convection from maintained and instantaneous sources. Proc. Roy. Soc. A 234, 1-23.

Papalliou, D. D. \& Lykoudis, P. S. 1974 Turbulent vortex streets and the entrainment mechanism of the turbulent wake. J. Fluid Mech. 62, 11-31.

Ricot, F. P. \& Spaldina, D. B. 1961 Measurements of entrainment by axisymmetrical turbulent jets. $J$. Fluid Mech. 11, 21-32.

Rouse, H., YiH, C. \& Humprreys, H. 1952 Gravitational convection from a boundary source. Tellus 4, 200-210.

Shadghnessy, E. 1975 Measurement of particle diffusion in a turbulent jet by laser light scattering. Ph.D. thesis, University of Virginia. 
Journal of Fluid Mechanics, Vol. 81, part 1

Plate 1

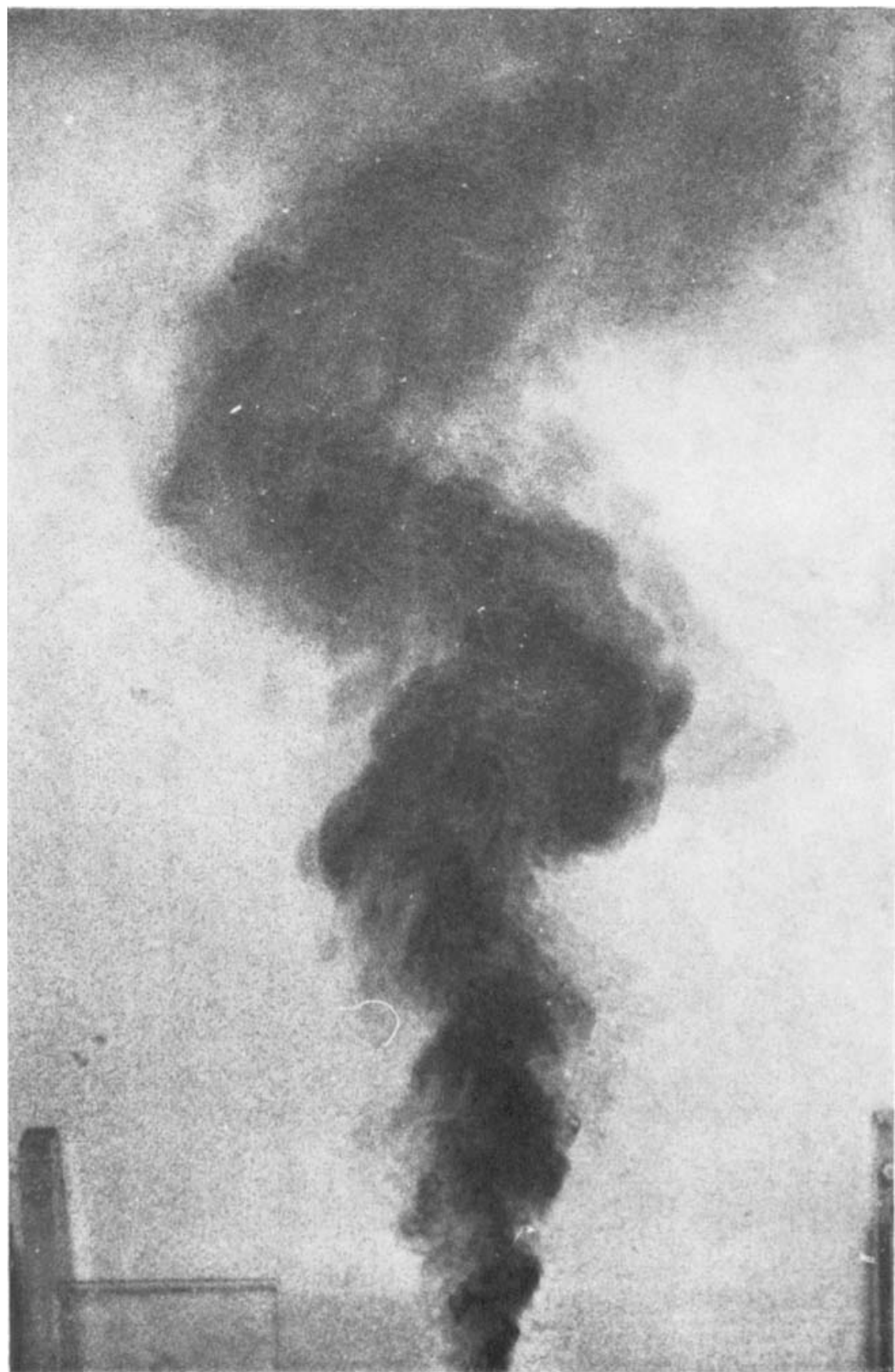

FigURE 18. Short time exposure $(0 \cdot 01 \mathrm{~s})$ photograph of a turbulent two-dimensional plume $\left(R_{0} \approx 0 \cdot 5\right)$. 


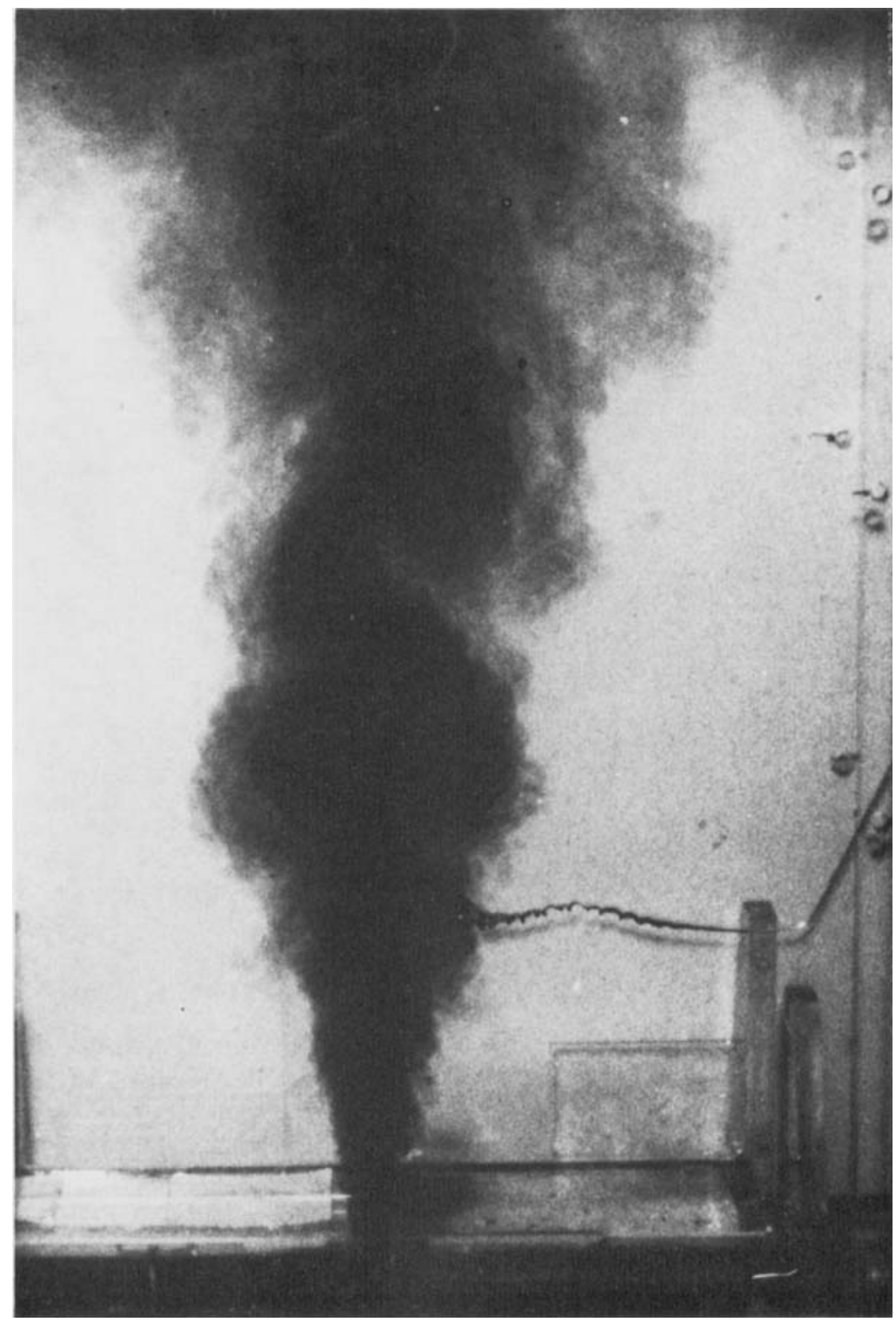

FrgUre 19. Medium time exposure ( $2 \mathrm{~s}$ ) photograph of a turbulent two-dimensional plume $\left(R_{0} \approx 0 \cdot 5\right)$.

\section{KO'TSOVINOS}




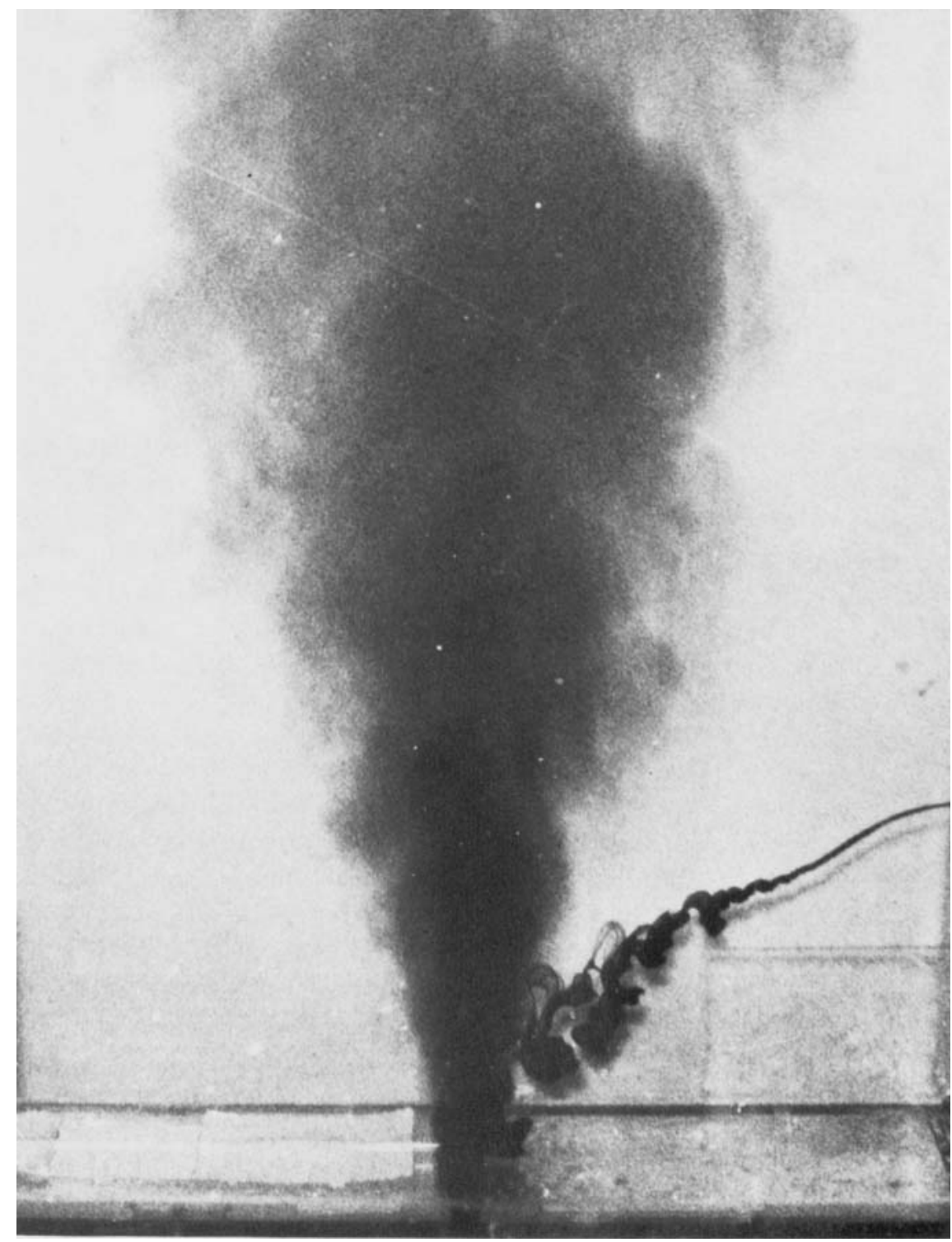

Figure 20. Medium time exposure (2s) photograph of a turbulent two-dimensional jet ( $R e \approx 2500, R_{\mathbf{0}}=0$ ). 